\title{
Complexity theory and the web
}

\section{Luke Tredinnick}

Department of Applied Social Sciences, London Metropolitan University, London, UK

Abstract

Purpose

- This paper aims to explore the influence of complexity theory on the development of the

web. It seeks to critique the role of complexity theory as a governing metaphor in the discourse of the web, and to examine whether complexity theory is able to provide an adequate description of the web, and its relationship to society and knowledge.

Design/methodology/approach

- The paper is a critial review.

Findings

- The paper establishes the influence of complexity in the discourse of the web and

questions the adequacy of complexity theory to provide a description of the web and its relationship to cognition and society.

Originality/value

- This paper explores the influence of a single concept (complexity theory) on the

discourse and development of the web.

Keywords

Complexity theory, Worldwide web, Society

Paper type

Conceptual paper

Introduction

This paper critiques the influence of complexity theory on the discourse of the worldwide web. It will argue that complexity theory has played a central role as a governing metaphor in the ongoing development of the web. The influence of complexity theory has led to the incorporation of largely unarticulated assumptions into the discourse of the web. By remaining largely unarticulated, those assumptions have avoided becoming subjected to proper scrutiny. This paper will therefore first summarise the field of complexity research and its uses in the social sciences. It will then examine how the idea of complexity emerges at two decisive moments in the web's development: its inception, and its movement to a Web 2.0 model. Finally it will explore the adequacy of complexity theory as a description of web technologies and their relationship to society, information and knowledge[1].

Adaptive complexity

Adaptive complexity describes the behaviour of large scale, highly dynamic systems such as the economy, the ecology, and society, each of which although driven by the aggregated behaviour of individual elements seems to function in coherent and motivated ways. The field of complexity research explores the characteristics and evolution of such systems, and seeks to explain their apparently motivated nature. It emerged out of the interdisciplinary Santa Fe Institute during the 1980s. There, researchers from mathematics, computing, the physical and social sciences were drawn together in an attempt to synthesise new ways of understanding adaptive complexity (Waldrop, 1992; Lewin, 1999). Complexity theory has therefore always occupied an interdisciplinary space. Although closely related to fractal geometry, catastrophe theory and chaos theory (Waldrop, 1992; Lewin, 1999), its heuristic outlook The current issue and full text archive of this journal is available at www.emeraldinsight.com/0022-0418.htm 


\section{Complexity \\ theory and \\ the web}

797

Received 10 September 2008

Revised 19 December 2008

Accepted 31 December 2008

Journal of Documentation

Vol. 65 No. 5, 2009

pp. $797-816$

$q$

Emerald Group Publishing Limited

0022-0418

DOI 10.1108/00220410910983119

was not limited to mathematical modelling. Instead it became a both a mode of analysis

and a series of ideas for describing the characteristics of certain kinds of systems.

Complexity research does not represent a unified field, but a loose and heterogeneous

collection of ideas, outlooks and methodologies that Thrift has described as an

"economy of concepts" (1999).

The concept of complexity is often defined in opposition to both complicatedness and simplicity (von Baeyer, 2003; Cilliers, 1998; Lewin, 1999). A system composed of many intricate parts is merely complicated unless the interaction of those parts is rich, dynamic, and non-linear leading to a change in their organisation over time (Cilliers, 1998; Manson, 2000). Thus a digital computer is merely complicated, being composed of many intricate parts that interact is a strictly linear fashion. A system crosses the threshold between simplicity and complexity when the number of such dynamic interactions reaches the point of system criticality, or self organised criticality (Manson, 2000), sometimes known as the tipping point (see Gladwell, 2002). Complexity therefore only reveals itself at a certain scale of observation; an individual financial transaction may be simple, but the aggregation of transactions within an economy becomes complex, driven by non-linear feedback. Cilliers (1998), argues that "the distinction between complex and simple often become a function of our 'distance' from the system" (p. 3); complexity only becomes "manifest at the level of the system itself" (p. 2). The complex system can therefore only be adequately described holistically.

It this holistic description of the behaviour and organisation of such systems on which the different strands of complexity research converge. Complexity research has tended to assume an anti-essentialist stance, emphasising the perceived naivety of scientific reductionism, and stressing that some qualities of systems are "not analytically traceable from the attributes of internal components" (Manson, 2000). Thrift has argued, "the chief impulse behind complexity theory is an anti-reductionist one" (1999). Urry (2005) has argued, "it is not that the sum is greater than the size of its parts - but that there are system effects that are different from the parts" (p. 5); in Anderson's (1972), pithier terms, "more is different". This opposition of complexity with reductionism acts both to contrast complexity theory with the general scientific tradition, but also through that contrast to reassert the scientific nature of the field. Complexity theory represents a re-calibration of what kinds of perspectives can be constituted as science. It provides what Thrift (1999), describes as a "semantic space" within "obdurate tradition", that "might allow us to think again about the world in more explicitly

spatial terms".

Fundamental to this spatial re-envisioning is the network or "meshwork" (De

Landa, 1997) of interlocked agents, motivations and forces that make up the complex 
system. Adaptive complex systems share the following characteristics (Cilliers, 1998, 2005; see Taylor, 2001; Manson, 2000):

They consist of a large number of individual elements.

Those elements interact dynamically (sharing information and exerting agency).

Those interactions are rich in nature. Each element will interact with many others.

Those interactions are non-linear leading to feedback. Positive feedback will tend to enhance, strengthen or stimulate interaction, and negative feedback will tend to inhibit, weaken or discourage interaction.

\section{JDOC}

65,5

798

Local interaction tends to predominate. Distant interaction is not impossible, but is inhibited by the network structure of the system. Global system effects will nevertheless tend to result from the aggregation of local interaction across the entire system.

They are open systems. They are affected by and affect their environment, tending to adapt to suit environmental conditions. The boundaries of complex systems are frequently indistinct, and often a matter of particular descriptions of those systems (see Manson, 2001).

They operate far from equilibrium. Complex systems are constantly adapting and evolving. They are never static, although they may be stable.

They therefore unfold in time. Time cannot be excluded from a description of a complex system. Both the current state and future evolution of a complex system are influenced by its history.

Each element of a complex system is ignorant of the whole and unmotivated by the global needs of the system, reacting merely to local conditions. The global organisation of the system is therefore unmotivated and emergent.

The complex system therefore forms a network or meshwork that is defined in terms of its relationships and which can be described in terms of information processing. This characteristic informs two key features of complexity: emergence and self-organising adaptation. Some qualities of adaptive complex systems only become apparent at the level of the system itself. Such qualities are termed emergent. For example, economic booms and recessions can be described as emergent qualities of the economic system, arising unmotivated as a result of the aggregated behaviour of individuals subject to positive and negative feedback effects. They are phenomena that only emerge at the level of the economic system itself, and cannot be understood purely in terms of the behaviour of individuals engaged in isolated financial transactions. Self-organising adaptation describes the tendency for complex systems to dynamically respond to changing internal and external pressures, resulting in their apparently motivated nature. Adaptation is sometimes manifested as a shift between stables states frequently described in terms of bifurcation or phase transition (De Landa, 1997; 
Waldrop, 1992; Lewin, 1999). It arises as a consequence of rich dynamic non-linear interaction subject to feedback, and therefore cannot be directly traced to the motivations or actions of individual elements of the system.

The most significant strand of complexity research within the social sciences has been that of aggregate complexity (Manson, 2000). The popularity of aggregate complexity within social theory derives from the kinds of descriptions it allows. Its models tend to be highly qualitative and holistic, resistant to the kinds of mathematic descriptions that dominate algorithmic and deterministic complexity (see Manson, 2000). It is worth tracing a relevant example to illustrate this. The social system can be understood as emerging in the dynamic and non-linear interaction of many independent agents: individuals living out their daily lives. Social interaction is generally local in nature, but is also very rich. We will tend to interact most with those immediately around us, but many such interactions occur between people. Social interaction is non-linear and asymmetrical; social bonds of different strength form over time, but however enduring or ephemeral are rarely entirely symmetrical. Society is an open system, and it is difficult to draw the boundaries between society and its

\section{Complexity \\ theory and}

\section{the web}

\section{9}

environment, between different societies, or between different sub-cultures within a society. Society operates far from equilibrium; the nature of society is constantly changing and mutating, sometimes through rapid upheavals, and sometimes through gradual evolution. It is therefore impossible to describe the socio-cultural system without reference to its history; society never returns to a previous state, and its history conditions the present and the future, but its future is nevertheless undetermined. Each individual contributes to the whole system, but remains ignorant of the whole in which they participate. Consequently the shape and evolution of society cannot be entirely controlled by individuals within it. Society, its history, and its evolution emerge out of the aggregation of behaviour across the entire system and therefore can only be described holistically.

While simplistic, this sketch of the complex social system illustrates the alluring power of complexity theory to provide apparently coherent and scientifically framed descriptions of diffuse social phenomena. It is this power that has led to the rise of complexity research within the social sciences (cf. Nowotny, 2005). Complexity theory has been applied to a wide range of social system, including the economy, society and culture (Waldrop, 1992; Lewin, 1999). Examples include: Taylor's synthesis of the cultural impact of digital technologies (2001); Fikentscher's model of culture and complexity (1998), and De Landa's non-linear social, economic and cultural history (1997). In addition, socio-cultural complexity has emerged through the field of meme theory, particularly in the work of Balkin (1998), Blackmore (1999), and De Landa (1997). The growing interest in complexity research in social theory has been described as the "complexity turn" (Urry, 2005).

In this paper it will be argued that complexity theory has had an important role in the development of the web, provided both a vocabulary and conceptual model for its description. This appeal to complexity has resulted in the incorporation of largely unarticulated and unanalysed assumptions within the discourse of the web in three specific areas: the organisation of society and culture, the ontology of information, and the web's underlying epistemology. The analysis in this paper will concentrate on two decisive moments in the web's history: its inception and its transition to a Web 2.0 
model. This is in part expedient: it would be impractical if it were even desirable or possible to trace the entire discourse of the web in a paper of this kind. But it is also strategic: these two moments bookend the development of the web from its inception to its re-emergence in Web 2.0. While the texts examined here are not representative of the entire discourse of the web, they are moments in that discourse that have nevertheless come to be regarded as significant in their own terms. Of the four texts from Berners-Lee discussed in this paper, two were written broadly contemporaneously with the creation of the web, and two at some distance from that time. There are important differences of perspective between these papers that relate to Berners-Lee's own changing relationship with his invention, but there is also much continuity of ideas between them. The texts used to illustrate Web 2.0 present fewer problems in this regard, appearing broadly contemporaneously.

Complexity at the inception of the web The influences on the development of the web are many and varied, and cannot be reduced to a simple application of complexity theory. Hypertext has its origins in Vannevar Bush's Memex machine. Bush cited "the artificiality of systems of indexing" JDOC

\section{5,5}

800

as the key problem in effective information retrieval (Bush, 1945; McKnight et al., 1991;

Woodhead, 1991). The word "hypertext" was coined by Ted Nelson, who sought to address what he perceived as a fundamental problem with traditional systems of indexing: not that they are intrinsically bad, but that different people at different times required different approaches (Naughton, 1999; Gillies and Calliau, 2000; Landow, 1997). Both Bush and Nelson implicitly reject analytical approaches to defining the relationships between individual documents, focussing instead on loose, contextual associations. The worldwide web was preceded by various implementations of a hypertext system, most notably Hypercard (Berners-Lee, 1999; Naughton, 1999). It was also preceded by various attempts to create a universal networked information system, including not only Nelson's Xanadu project, but also the menu-driven Gopher system (Berners-Lee, 1999; Naughton, 1999; Gillies and Calliau, 2000). In different places Berners-Lee $(1990,1995,1999)$ and Berners-Lee (1992

et al.), has acknowledged the influence of all these factors on the creation of the worldwide web. However, it is also clear that complexity theory played a key role in Berners-Lee's work. Indeed, the attraction of hypertext may derive from the way in which it superficially appears to model complex relationships. It is also complexity theory that came to mark-out key differences between the structure of the web and that of earlier hypertext systems. It is well known that the web was developed at the European Organization for Nuclear Research (CERN) during the late 1980s and early 1990s (Berners-Lee, 1999; Naughton, 1999; Gillies and Calliau, 2000). The idea of a distributed hypertext system was well developed before the first proposal was circulated within CERN (Berners-Lee, 1999). However, in that original proposal the system was already being framed in terms of self-organising adaptive complexity. The opening lines announce the scope of the project in the following way:

This proposal concerns the management of general information about accelerators and experiments at CERN. It discusses the problems of loss of information about complex evolving systems and derives a solution based on a distributed hypertext system (Berners-Lee, 1990). 
The allusion to "complex evolving systems" invokes the emerging field of complexity research. At the moment of its first articulation, complexity defines the problem that the web aspires to address. It will later also comes to define the solution.

On face value the complex evolving systems here are merely those research projects within CERN about which there is a tendency for information loss. This anxiety about the effective management of information pertaining to ongoing research reflects in part the complicated working structure at CERN (Berners-Lee, 1999; Gillies and Calliau, 2000; Naughton, 1999). However, it becomes clear that the phrase complex evolving systems provides for Berners-Lee not only a model of the characteristics of individual research projects, but also of CERN itself and its working practices. He describes CERN's "observable working structure" as "a multiply connected 'web' whose interconnections evolve with time" (1990). The meshwork or network in constant flux, central to adaptive complexity, emerges as a ready metaphor for social organisation.

His description of the cascading effects of change (a topological reorganisation of the meshwork) also hints at the influence of complexity research. Change filters through the organisation: "when a change is necessary [

...] one has to dig around to find out

what other parts and people will be affected" (1990). In later writing Berners-Lee (1999)

reflects on CERN's "weblike structure" (p. 10) and "complexity" (p. 15), and notes that

Complexity

theory and

the web

801

CERN is really "an extended community of people who have relatively little common authority" (p. 9). These different descriptions echo key themes of complexity research: network structures defined in terms of relationships rather than nodes; the evolution of structure over time; the distributed nature of agency; the self-similarity of structure throughout the system, and the whole emerging in the aggregated behaviour of its constituent parts (Cilliers, 1998, 2005; Manson, 2000). It is striking how consistently CERN is described in these terms throughout Berners-Lee's work.

While only directly applied to the working environment and governance of a single organisation, there are two reasons for thinking complexity provided a more general metaphor for social organisation in Berners-Lee's work. First, CERN is explicitly situated as both an extreme case of social organisation in general, and as a fulfilment of a general trend towards more networked social structures. Berners-Lee argues, "CERN is a model in miniature of the rest of world in a few years time. CERN meets now some problems, which the rest of the world will have to face soon" (Berners-Lee, 1990). The situation at CERN both exemplifies the information management issues facing the world, and can be regarded as a model for addressing those issues; the perceived self-similarity between the organisation and its wider social context organises the relationship between the two. Second, the web is explicitly situated in relation to both its local and global context. It is already clear in the original proposal that the web was intended to address more than parochial concerns. Berners-Lee described it as "a universal linked information system" (1990); its universality emphasised by the open protocols and standards at the heart of the project (Berners-Lee, 1999). The name "worldwide web" chosen before it could properly be described as either worldwide or a web, encloses its aspiration to universality. It is an aspiration to the outside, to the global social context, to the worldwide problems of information management in the digital age. By 1992 that aspiration was been stated in more confident and concrete terms: the web is "a practical project designed to bring a global information universe 
into existence using available technology" (Berners-Lee

et al., 1992). To be a universal

solution, the web must be understood to be addressing universal problems. Because

those problems are defined in terms of complexity, complexity becomes insinuated into the description of the wider social context.

While social complexity remains largely in the background of the original proposal, never formally articulated, it becomes more explicit in Berners-Lee's later reflection of the development of the web. In 1995 he wrote:

A few years ago, the world was fascinated (quite rightly) with fractal patterns [

...] Is society

fractal? Yes, it certainly is. There is structure at the highest levels and the lowest levels. There

are great big links formed by organizations which themselves are made up of smaller links.

You can simplify society on a number of levels [

...] People need to be part of the fractal

pattern. They need to be part of organisms at each scale. We appreciate that a person needs a

balance between interest in self, family, town, state and planet. A person needs connections at

each scale. People who lack connections at any given scale feel frustrated (Berners-Lee, 1995).

Fractal geometry and complexity theory are closely related fields. Therefore although clothed in the metaphor of the fractal, this is also a paradigmatic description of society as a complex adaptive system. It invokes all the conditions for complexity set out by Cilliers $(1998,2005)$. Berners-Lee also implies psychological motivations: needs, and frustrations, and self-interest. It is the psychological explanation of social organisation that in many ways aggregate complexity theory seeks to overcome. In this extract they JDOC

\section{5,5}

802

bridge the gulf between mathematical metaphor and its realisation in the social world. Nevertheless, psychological motivations are never the cause of self-organisation in his description of society. They are the outcome of an imposition on, or frustration of society's innate self-organising potential.

Throughout his writing about the web and his reflection on its development, Berners-Lee alludes to the complex nature of social interaction in a number of different ways. The social system is a network, defined in terms of the relationships between individuals. Those relationships are dynamic and evolving, leading to a distribution of influence and authority across the network. Interaction is driven by small-scale rules that operate at the local level, but which result in global effects and large-scale structures. Social agency and influence tends to cascade through the system. The social system exhibits self-similarity, with the whole resembling the parts out of which it is composed (family, town, state and CERN itself). While never explicitly stated, it is clear that complexity theory played an important role in framing the problems of information management that the web was intended to address. Complexity both becomes the cause of those problems, and demonstrates the inadequacy of existing information management solutions whose strictly hierarchical structure cannot be adequately mapped onto the constantly evolving social context.

If complexity becomes a description of the problems of information management within a dynamic social system, it also becomes a description of the solution. The evolving network becomes a way of addressing the problem of information management in an unstable, evolving social context. The structure of the web mirrors the evolving social structures within which it is embedded. Berners-Lee writes:

When describing a complex system, many people resort to diagrams with circles and arrows. Circles and arrows leave one free to describe the interrelationships between things in a way that tables, for example, do not. The system we need is like a diagram of circles and arrows, 
where circles and arrows can stand for anything (Berners-Lee, 1990).

This is a description already beginning to converge on the RDF metadata standard.

Entities and connections are allowed here stand for anything, as they can in RDF, but not in HTML (where they relate to documents and hyperlinks respectively). Two important ideas about information and its structure emerge in this description: its network structure, and the location of value in network connections rather than nodes. Those connections define relationships, and it is relationships that describe the ontology of the web. Within complexity theory relationships are not a matter of physical connections, but of information transmission or agency. The complexity of the meshwork is therefore not only an ontological description, but also a description of the process of information transmission and agency over time. Berners-Lee's account of the linked information system converges on this kind of model: information is defined by its relationships, but it is the relationship, connections, or links that fully embody the idea of the global or universal information system. They embody that idea because they enable self-organisation. They enable the system to respond to changing environmental conditions. The value of information that emerges from this description is precisely located in its ongoing repositioning in relation to the entire information system. The agency of information, what we might think of as the meaning of information (see Tredinnick, 2006a), is located in those ever changing connections and associations that in the web become externalised in the idea of the hyperlink. Just preceding the previously cited quotation, Berners-Lee writes:

\section{Complexity theory and the web \\ 803}

In providing a system for manipulating this sort of information, the hope would be to allow a pool of information to develop which could grow and evolve with the organisation and the projects it describes. For this to be possible, the method of storage must not place its own restraints on the information. This is why a "web" of notes with links (like references) between them is far more useful than a fixed hierarchical system (1990).

The perceived self-organisation potential of the web is clearly articulated here. But the idea of "information" also slips between its general and special meanings: information as document, and information as a form of agency (later, the "topology of information" (1992)). Manipulation refers to both the nodes and the relationships between them. It is this complex self-organising characteristic of the web that allows it to mirror the place of information within the complex socio-cultural system. Through its mirroring action, its redoubling of the complex social system, the linked information system overcomes limitations that are implicit in other kinds of approaches where structure is imposed, rather than emergent. In a later paper, Berners-Lee notes "The web stretches seamlessly from small personal notes on the local workstation to large databases on other continents" (Berners-Lee et al., 1992). It stretches seamlessly between different scales precisely because of the self-similarity of the part to the whole. The fractal nature of society is also a fractal description of the networked information system. The web is as a result more useful than fixed hierarchical systems, which represent "less flexible ways of finding information" (1990). While it remains questionable whether the forging of links between documents is not also an imposition of structure, the distinction is between pre-existing formal structures, and emergent structures that evolve over time. It is in this sense that the web never imposes its structure, but is rather always inside information itself, always inside the pre-existing relationships that already define information within a social context. It is the embodiment of the idea 
of emergence.

If the web reaches out to the complex nature of the socio-cultural system within which information is deployed, and through which information is always embodied in its meaning and use, then it also reaches inwards to mirror the subjective experience of information and meaning. In 1992 Berners-Lee reiterated the self-organising nature of the web, but this was now a self-organisation that also reflected the structure of knowledge: "links allow the topology of the information to evolve, so modelling the state of human knowledge at any time is without constraint (Berners-Lee et al., 1992).

Unmentioned in the original proposal, knowledge becomes the heart of Berners-Lee's later descriptions of the web. In the same paper, Berners-Lee notes: Computers give us two practical techniques for human-knowledge interface. One is hypertext, in which links between pieces of text (or other media) mimic human association of ideas. The other is text retrieval, which allows associates to be deduced from the content of text (Berners-Lee

et al., 1992).

The one is emergent, the other analytical. The one is represented by rich-media, the other only by text. The one arises out of knowledge as it is embodied in human minds, the other out of knowledge as it is embodied in text. Hypertext

mimics the human association of ideas, where text retrieval merely mimics the text, and hypertext hence becomes a model of the state of human knowledge that is without constraint, where text retrieval is only a model of explicit knowledge constrained by the nature of the document. Two different conceptions of knowledge are at work in these last two

\section{JDOC}

\section{5,5}

\section{4}

extracts: one tacit ("human association of ideas") and one explicit ("state of human-knowledge"). They are conflated in this description, entangled by an association of ideas: the redoubling of each in the image of complex web. The state of human knowledge becomes the aggregation of individuals' association of ideas scaled-up across the entire system. Explicit knowledge becomes particular concrete articulations of "the state of human knowledge"; concrete articulations that are constantly redefined, reconstructed, resituated, and mediated by the complex web, and whose status is therefore in constant flux.

Superficially these statements seem to articulate an epistemological model, but it becomes clear they reflect something more radical. In 1999 Berners-Lee suggested:

In an extreme view, the world can be seen as only connections, nothing else. We think of a dictionary as the repository of meaning, but it defines words only in terms of other words. I liked the idea that a piece of information is really defined only by what it's related to and how it is related. There is really little else to meaning. The structure is everything. There are billions of neurons in our brain, but what are neurons? Just cells. The brain has no knowledge until connections are made between neurons. All that we know, all that we are, comes from the way in which neurons are connected (Berners-Lee, 1999, p. 14).

Despite their implications, these comments have received relatively little critical attention. The cautiousness of the opening gambit - "in an extreme view" - both undermines what follows, and is undermined by preceding the only view given. If it is extreme, it is also entirely consistent with the ontology of complexity. In this extreme view, the world, language, information, cognition and knowledge are all only connections. They are all merely nodes in the complex web, defined entirely by their relationships. Knowledge is reduced to a formal relationship between things neurons in an outlook that echoes Hebb's model of synaptic reinforcement (see Gardner, 1987; 
Penrose, 1989), itself an important influence on cognitive complexity research (see Cilliers, 1998); The idea of meaning that emerges in this description is not the thing-in-itself, but the thing-in-relation: the thing as it is held in the web of association of ideas, neurons, and of course people.

It is striking how close Berners-Lee comes here to post-structuralist theory, particularly the work of Derrida. The connections that bind the ubiquitous web are similar to the play of diffe' rance in Derrida's work (1976). Meaning is for Berners-Lee as for Derrida situated within the trace, never fully embodied, and always slipping out of view (1976). It is striking perhaps, but not surprising. Cilliers has delineated the close conceptual similarities between complexity theory and post-structuralism (1998; 2005); both are broadly speaking predicated on an anti-essentialist rejection of the analytical mode. That it is complexity not post-structuralism that provides the touchstone of this statement is confirmed by Berners-Lee's later acknowledgement that his "may be a view influenced too much by physics" (1995). It may well be, but it is nevertheless the physics of dynamic non-linear systems theory rather than classical mechanics. In the theoretical foundation of the web, complexity theory therefore insinuates itself on three levels: social organisation, information, and knowledge. But complexity theory does more than provide a vocabulary and metaphor. The web's architecture progressively aspires to reproduce the complexity it models in a number of ways that explicitly set it apart from earlier implementations of hypertext. Where Nelson had insisted on bidirectional hyperlinks, only unidirectional hyperlinks were integrated into the architecture of the web. This may in part merely reflect the practical issue of

\section{Complexity}

\section{theory and}

the web

\section{5}

implementing a distributed hypertext system (Berners-Lee, 1999), but it also suggests how the architecture of the web is always aspiring to reproduce its own complexity. Unidirectional hyperlinks are a precondition of the web's aspiration to adaptive complexity, making possible the emergence of asymmetrical relationships that are fundamental to self-organisation. In addition, the web has converged on the model of complexity throughout its development. The extensible nature of XML reincorporates emergent structure within the document itself where it had been banished by the reserved terms of HTML. The structure of RDF more fully embodies aggregate complexity defined through relationships rather than entities, converging on the idea that "the world is only connections" (Berners-Lee, 1999). The progressive movement highlights how complexity theory provided more than a set of governing metaphors for the ontology of the web. It became an ongoing agenda in its development, an agenda that marks out key philosophical distinctions between the web and earlier implementations of hypertext.

Complexity and Web 2.0

If we turn from the web's inception to more recent developments, it is not to imply that complexity theory played no role in the intervening period; developments like the semantic web embodied in RDF and XML imply otherwise. Rather by turning to the idea of Web 2.0 the continuing centrality of complexity theory to the discourse of the web will be suggested. Although a term possessing some previous currency, it is O'Reilly's conception of Web 2.0 that has in recent years come to dominate the discourse of the web (2005). The usefulness, relevance and clarity of his model of Web 2.0 remain controversial (see: Tredinnick, 2006b, c; 2008). However, this paper will not address that controversy directly, exploring only how O'Reilly and others both 
re-iterate and re-articulate ideas drawn from complexity theory. While less prone to direct comments on the nature of society and knowledge, the discourse of Web 2.0 follows many of the themes already present in Berners-Lee's work. The inculcation of complexity is therefore less systematic and apparent, but it is no less potent for it. O'Reilly's description of the new web is initially nebulous and difficult to relate to concrete applications or services. Its characteristics are established in series of oppositions that exemplify the old and new approaches: DoubleClick/AdSense; Britannica/Wikipedia; publishing/participation; stickiness/syndication, and so on. In each, the idea of Web 2.0 is carried not in particular applications and services that mark out a perceived shift, but in the differences between them. It is not that Wikipedia, for example, embodies the idea of Web 2.0, but rather that Wikipedia embodies a difference in attitudes and outlook that differentiates it from Britannica and which associates it with blogs, syndicated content, folksonomies and BitTorrent. This conceit allows Web 2.0 to be defined predominantly in terms of what it is not. It is not a particular set of applications (wiki, or blog), nor a particular set of services (Google or Amazon), nor a particular set of information management techniques (folksonomies, or social bookmarks). Web 2.0 is merely "an attitude" that governs the development of information services (O'Reilly, 2005). That attitude is centred on valorising the emergent qualities of web services. But the definition of Web 2.0 in a series of differences also constructs the idea of Web 2.0 itself as a series of relationships that draws on the metaphor of the meshwork or network. It is the system itself rather than the nodes out of which it is constructed that define the idea of Web 2.0. O'Reilly's JDOC

\section{5,5}

\section{6}

refusal to reduce the idea of Web 2.0 to the concrete and the particular has fuelled perceptions of its meaninglessness (Shaw, 2005; Anderson, 2006). Yet this refusal can also be seen as a strategic emphasis of the whole over its parts. It helps construct for the new web what Thrift described as a "semantic space" that "might allow us to think again about the world in more explicitly

spatial terms" (1999).

O'Reilly twice introduces meme theory to support and elaborate the perceived shift in attitudes that is marked out by the new web. "Web 2.0" is both a meme and a concept composed of memes. On one level "meme" functions merely as a vogue-word with some existing currency within computing subculture used only to emphasise the supposedly radically different attitude of the new web. But it also situates O'Reilly's paper quite precisely in relation to both the epistemology of Web 2.0 and the social structures in which it sits. It tacitly articulates a particular theoretical position that while announcing itself fails to fully engage in its own consequences. Memes originate in the work of Richard Dawkins $(1976 ; 1983)$. They represent the cultural equivalent of genes: self-replicating units of selection that operate among other memes in a meme-pool, and combine in memeplexes to form complex cultural formations such literature, art, music and traditions. Although intended to illustrate genetics (Dawkins, 1999), meme theory was later transformed into a description of cultural evolution (Balkin, 1998; Blackmore, 1999; De Landa, 1997). In this movement the meme was made into an articulation and embodiment of socio-cultural complexity. Meme theory conceives socio-cultural change as en evolutionary process governed by natural selection. Memes are dependent on minds for their propagation, but are independent of the vehicles of their transmission. The meme never exists as a material thing, but exists only in it its influence and agency: the way in which it changes the state of the mind into which it is 
introduced so as to encourage its own replication. The meme is therefore a principle of the transmission of agency in the same sense as information within complexity theory. By introducing the idea of the meme to describe Web 2.0, O'Reilly (2005) implies a particular view of social and cultural organisation and self-regulation. It is an idea that echoes Berners-Lee's comment that "the world can be seen as only connections" (p. 14). The meme reinforces the description of Web 2.0 as a network of relationships. This view becomes explicit in the conceptualisation of Web 2.0 as a family of memes, many of which echo ideas drawn from complexity research. The long tale explicitly alludes to the power laws that govern many complex systems. The idea of the perpetual beta alludes to the adaptive nature of complexity and their resistance to final stable states. The idea of granular and composite services reflects the basic network structures at the heart of complex systems. Most significantly, the idea of emergence haunts O'Reilly's description of Web 2.0, surfacing in phrases such as "hackability", "software that gets better the more people use it", "emergent user behaviour not predetermined", "play", "harnessing collective intelligence" and "trust your users" (O'Reilly, 2005). Each of these ideas develops a key theme of Web 2.0: that aggregated user behaviour provides a mechanism to unleash the potential for the self-organising adaptation of web services. Other writers on Web 2.0 also echo the centrality of emergence. Miller (2005) for example stresses "ad hoc relationships" that allow users to "combine and recombine that which they are given in new and interesting ways". Sterling (2005) has stressed the spontaneous nature of folksonomy structures emerging through aggregated behaviour. O'Reilly (2005) himself argues that "network effects from user contributions are the key to market dominance in the Web Complexity

theory and

the web

\section{7}

2.0 era". Many of the technologies associated with Web 2.0 incorporate the idea of emergent structure: the folksonomy, blog, social bookmarking, and wiki exploit "the wisdom of crowds" (O'Reilly, 2005; see Surowiecki, 2004), epitomising the idea of an information system that evolves in response to the actions and needs of its users. In its attempt to capitalise on user behaviour, Web 2.0 therefore exemplifies Anderson's (1972) pithy description of a system in which "more is different".

The information web becomes responsive to the emerging patterns of the complex socio-cultural system precisely because it allows those system effects to be redoubled by the web through the aggregation of user behaviour and contributions. The mental-map of the web these ideas articulate is identical that of Berners-Lee; it is not just a web, but a "complex evolving system" with a differential structure. The complex self-organising nature of the web is therefore also a description of the socio-cultural system that houses it; in the discourse of Web 2.0 the two are difficult to separate.

O'Reilly (2005) argues:

Much like a phone call, which happens not just on the phones at either end of the call, but on the network in between, Google happens in the space between browser and search engine and destination content server.

The spaces between are occupied by relationships, and the meaning of information just as for Berners-Lee is defined by those relationships, rather than by the content of nodes. But critically those relationships are formed not only between web content, but also between users. O'Reilly notes: "like Wikipedia, blogging harnesses collective intelligence as a kind of filter" (2005); collective intelligence like the "wisdom of crowds" (O’Reilly, 2005; see Surowiecki, 2004) relates not to the evolving web per se, 
but to the complex socio-cultural interaction that both pre-exists the web and drives its evolution. The web is therefore a "platform" for social interaction and participation (O'Reilly, 2005). In Vander Wal's (2007) terms this is not a matter of categorising information, but of "providing a means to connect items [

...] to provide their meaning".

Items are connected on the basis of social relationships. The value of information already exists in the relationships that are embodied by, and which pre-exist the web:

There's an implicit "architecture of participation", a built-in ethic of cooperation, in which the service acts primarily as an intelligent broker, connecting the edges to each other and harnessing the power of the users themselves (O'Reilly, 2005).

The network is not only the web, but also the socio-cultural system into which it is integrated. Services become the "broker" of modes of social participation and communication, "connecting the edges" not just of the digital network, but also of the social network (O'Reilly, 2005). O'Reilly (2005) argues that services must: "leverage customer-self service and algorithmic data management to reach out to the entire web, to the edges and not just the center, to the long tail and not just the head". This echoes Berners-Lee's aspiration for a web that "stretches seamlessly from small personal notes on the local workstation to large databases on other continents" (Berners-Lee et al.,

1992), but the "long tail" also draws explicitly on the power laws governing the behaviour of many complex systems.

O'Reilly therefore echoes Berners-Lee's description of a web not only reaching outwards towards the social structures within which it is situated and into which it is integrated. He also echoes Berners-Lee's description of a web reaching inwards

\section{JDOC}

\section{5,5}

\section{8}

towards cognitive processes, although as with the description of society, the boundaries are more blurred. He writes:

Much as synapses form in the brain, with associations becoming stronger through repetition or intensity, the web of connections grows organically as an output of the collective activity of all web users (2005).

Web 2.0 reaches out to infiltrate the entire social system, and reaches in to infiltrate our cognitive process. It even (symbolically) replaces our internal dialogue with a shared social dialogue ("the blogosphere is the equivalent of constant mental chatter in the forebrain, the voice we hear in all of our heads" (O'Reilly, 2005)). It integrates a complex of complex systems: self, society, and self in relation to society.

While the discourse of Web 2.0 is perhaps more radical in its description of the web, there is also real a continuity with Berners-Lee's work. It is a continuity forged by situating the web as a mirror of both the complex society and the complex mind, and as a manifestation of complexity in its own right. That continuity is critical. Berners-Lee has commented:

If Web 2.0 for you is blogs and wikis, then that is people to people. But that was what the Web was supposed to be all along (DeveloperWorks, 2006).

If the first stage of the web's development broadly resulted in a publishing model resistant to user participation and adaptive self-organisation, driven out of the computing sub-culture by its rapid commodification, then its second stage perhaps represents the re-embodiment of an original aspiration of self-organising adaptive complexity. The idea of Web 2.0 is therefore only a return to a description of the web as the embodiment of complex self-organisation. Indeed O'Reilly explicitly acknowledges that "the '2.0-ness' is not something new, but rather a fuller realization of the true 
potential of the web platform" (2005).Web 2.0 is always already there within the idea of the web waiting to be unleashed; it is always already a becoming of that embodiment of complexity. Thus Web 2.0 does not begin with O'Reilly's paper, but is already a becoming of Berners-Lee's ideal of a self-organising system from the inception of the web. If Google, Wikipedia and Amazon already embody Web 2.0 in O'Reilly's paper, it is because Web 2.0 is an idea that finds its name only when it reaches criticality, reaches its tipping point, and becomes a dominant mode of user participation. The complex web finds its own justification in the criticality threshold of self-organisation. The adequacy of complexity theory It has been argued that complexity theory insinuated itself into the theory of the web from its inception on three levels: the complexity of the linked information system, the complexity of the minds that it mirrors in its use, and the complexity of the society that it mirrors in its ongoing creation. In doing so it not only provided a model for describing the web, but also an agenda for its ongoing development. A question remains as to why complexity theory played this central role in the discourse of the web. One reason is perhaps the close association between complexity research and computing in the eighties and nineties. Computer modelling became central to complexity research from its inception, exemplified by Langton's adaptation of Conway's Game of Life (see Gardner, 1987) and Kauffman's modelling of neural network interactions (Waldrop, 1992; Lewin, 1999). In the late 1980s, themes of

\section{Complexity}

theory and

the web

809

complexity and computing converged in the fields of machine intelligence, information processing, information theory and neural networking. To be thinking about some of the paradigmatic problems of computing at this time was also to be thinking about complexity. It is therefore on one level unsurprising that the discourse of the web later drew on some of the technical vocabulary and mental models of complexity research. However, there is perhaps also a political dimension to the inculcation of complexity theory into the discourse of the web. The idea of "order out of chaos" (Sterling, 2005; see Waldrop, 1992) reflects the liberal-libertarianism of the computing subculture, particularly in the USA where through Usenet, Bulletin Board Services, Free Software and the Open Source movement, that subculture began to articulate its own values in the late 1980s. The personal computing revolution was the child of the Woodstock generation (Roszak, 1988; Taylor, 2001), and echoes of 1960s the counter-culture can be seen in the hobbyist roots of the computing sub-culture. The ideals of this movement occasionally starkly echo the discourse of the web; Community Memory, an early bulletin board system, was described by one of its creators in the 1970s as:

An actively open ("free") information systems, enabling direct communication among its users with no centralized editing of or control over the information exchanged [

....] it

democratizes information-power, for no group of its users has more access to the main information than the least user has (cited by Roszak, 1988, p. 163).

Complexity theory was perhaps an idea that surfaced at a time, and within a community that allowed it to incorporate these political ideals into formal descriptions that concealed their political bite. It became more than a set of mathematic models, but a way of subtly articulating resistance to various forms of authority. It became perhaps a means of articulating the value of the social network itself, as opposed to the rules and controls imposed on it, and of insisting on the transcendence of the system, 
individual values, and individual action over those social controls, norms, roles and conventions that had tended to marginalise the computing subculture in its geek ghetto.

But it is unclear that complexity theory is adequate to this kind of description. It is tempting to see in it a sleight-of-hand: an attempt to conceal those political situated challenges and assumptions. A first sense of this emerges in the nar"ve and superficial application of cognitive science in the purported parallel between the structure of the web and the structure of cognitive processes. The dominant model of cognition is the computational model, in which the brain functions much like a neural network to process information (for example, Pinker (1994, 1997); Fodor (1976)). This generally assumes the validity of Hebb's conjecture about the reinforcement of synaptic connections over time (Gardner, 1987; Penrose, 1989), which provides a foundation for the complexity of brain functioning and neural network computing (see Penrose, 1989; Cilliers, 1998), and to which both Berners-Lee (1999) and O'Reilly (2005) allude. However, the computational model of cognition is by no means universally accepted (Penrose, 1989, 1995; Jakendoff, 2001), and Hebb's conjecture falls short of providing a full description of cognition. On this point Gardner (1987) has noted wryly that "the rigorous application of methods and models drawn from computational realm has helped scientists to understand the ways in which human beings are not much like these prototypal computers" (p. 44). The correlation between the structure of cognition and the structure of the web is therefore artificial. At best, the web mirrors only a part of what contributes to cognition.

\section{JDOC}

\section{5,5}

\section{0}

The tacit assumption incorporated into the discourse of the web is that the loose association of ideas "reflect more nearly the way in which the human mind thinks" (Ellis, 1992). Ellis (1992), has observed that this "may be based on a rather nar"ve concept of mind", and it is difficult to disagree. But even taken at this level, the consequences of the assumed correlation remain unclear. A superficial resemblance between the web and the brain underpins an assumption that hypertext allows a more natural mode of engagement unconstrained by the limitations of formal analytical classification. But there is a gulf between these two proposition filled only with innuendo. The structure of cognition contributes little to an understanding of the structures of conscious experience and behaviour, but it is on the level of conscious experience that users engage with information systems. Some people may therefore "resort to diagrams of circles and arrows" (Berners-Lee, 1990), but others equally resort to hierarchies and lists. Conscious thoughts may be "only connections", but those connections can be of any number of different kinds in addition to the "coveted random associations" valorised in the structure of the web (Berners-Lee, 1999, p. 11). Indeed, formal hierarchies and taxonomies have tended to proliferate across the web, because of the usability problems that loose, random and contextual associations pose. There is therefore no very clear reason to accept that networks provide a less constrained means of engaging with and organising information. They represent merely another kind of structure imposed on information. This does not imply that hypertext has no beneficial uses, but it does undermine the grounds on which that usefulness is justified within the discourse of the web. Complexity theory fails to provide the necessary connection between the structure of cognition and that of the information system.

A similar conflation occurs at the level of the complex social system and its relationship with the web. That society is a complex adaptive system is both true and 
at the same time largely unrevealing: aggregate complexity was derived from systems like society, and it is therefore unsurprising that it subsequently describes those systems in its own terms. It does not follow that complexity provides an adequate description: that it exhausts that description of society. Nor does it follow that complexity is adequate for describing the relationship between society and the web. Again, these are categorically different propositions. There is no reason to suppose that the web can capture the complexity of the social system merely by modelling its own form of complexity, and no clear justification is provided for supposing so. In one sense this reveals a basic naivety in the discourse of the web: a tendency to assume a dependent relationship on the basis of a mere correlation or similarity. But there is also a danger that complexity functions to conceal aspects of the relationship between the information system and society that we should want to be clearly exposed.

Complexity theory can only be usefully applied if it reveals relationships and patterns that would otherwise be obscured. But models of aggregate complexity often possess only descriptive and not explanatory power (Tredinnick, 2006a). That is to say that they often provide little more than superficially scientific descriptions of social processes, contributing little to our understanding of individual experiences. Indeed, they tend to exclude much that we might think central to our social experience, including motivations, emotions and desires. In fact the gesture of complexity conceals behinds its generosity towards the individual a mean-spirited outlook on individual agency. By conjecturing their emergence through social processes, it denies institutions, traditions and social structures power over the individual. But at the Complexity theory and the web

\section{1}

same time it denies the individual political power and social agency over institutions, traditions and social structures. The aggregate of individual behaviour is in the same movement a disempowerment of the individual as we each become engulfed by the tide of emergence. Thus, complexity theory conceals a starkly functionalist outlook that focuses strictly on outcomes and not on how they are achieved.

By drawing on complexity theory, the discourse of the web excludes many aspects of social experience that we might thing integral to the social role of the linked information system. Neither Berners-Lee nor O'Reilly are directly concerned with the individual experience of participation, nor with the social context in which that experienced is formed, but the constraints of the system on individual participation are crucial to any description of a complex society. The social system within which information is disseminated is always already structured in terms of class, ethnicity, educational levels, membership of particular groups, the pre-existing institutions and organisation that act as gate-holders to participation, and so on. The discourse of the web has tended to overlook one key idea from complexity theory: that the history of a system conditions its present, and that therefore its history cannot be excluded from the description of that system. Far from escaping the

a priori structures of knowledge organisation, the web tends to reinforce the differential structures etched into discourse. Not only is this reflected in the relative visibility of different languages on the web (Paolillo 
et al., 2005)) but also in the way in which forms of language already pre-condition, model and mould expression, influencing group membership, identity and participation (Tredinnick, 2008). Berners-Lee and O'Reilly neither address the politics of participation, nor how differential participation may undermine or subvert the self-organising potential of the web. In this refusal to engage with overtly political questions deriving from social experience that the discourse of the web converges on an apparently apolitical outlook. But by remaining unnamed, the power exerted by existing social formations in the perpetuation and creation of real inequalities of access and control is disarmed. By appealing to emergence the discourse of the web tends to obscure questions about the basis on which participation is established.

The incorporation of complexity into the discourse of the web therefore on one level provides only a self-fulfilling kind of description. That order emerges in the web through the aggregate behaviour of individual agents is self-evidently true. But it is a superficial and unrevealing truth. Structural order is also the pre-condition of social participation and interaction, and it is the interaction between these pre-existing structures and the information system that conditions what kinds of structural order emerges in the web. By concealing the social context of interaction, the discourse of the web allows little room for its critique. Aspiring only to merely mirror social organisation the web condemns itself to become saturated by the same power relationships that dominate the age of print and the age of media (Tredinnick, 2008). In the process it becomes locked into a set of doggedly subjective positions that cannot hope to adequately address the relationship between the web and the wider social system. It is for this reason that "the wisdom of the crowd" (Surowiecki, 2004) degenerates into "the rule of the mob" (Keen, 2007, p. 44). The two statements differ only in their subjective position in relation to emergent qualities. The discourse of the web is forced into binary extremes of this kind precisely because it doggedly ignores the role of the system's history in determining the processes of emergence. It cannot escape the influence of existing structures that it nevertheless refuses to name. Thus

\section{JDOC}

\section{5,5}

\section{2}

the discourse of the web tends to become locked in a largely false argument between overly optimistic voices centred on its democratising and empowering potential, and overly pessimistic voices concerned with its role in cultural decline and cultural relativism. Caught between these two extremes, the discourse of the web tends to perpetuate, conceal, and deny the potent role of the already existing structure of society, culture and discourse that in themselves already differentiate opportunities for participation.

If complexity theory poses more questions than it answers when applied to social organisation, then it also poses questions for its manifestation in the architecture of the web. The existing structures that condition the interaction of individual agents within the complex system also disrupt the assumed complexity of the web itself. The digital technologies on which we use web applications and services are always already structuring, delimiting, and mediating our use of those applications and services. TCP/IP, HTTP, FTP, HTML, Unicode and text are always already an imposition of structure. Google's search ranking and the folksonomy may both depend on the independent behaviour or individuals in their use of the web, but the algorithms on which they depend are already delimiting and structuring that behaviour within pre-existing assumptions about the relative preponderance of different qualities of that behaviour. Web 2.0 may allow the connections between information to be strengthened 
over time, but assumptions about how that behaviour is interpreted are already within the algorithms that perform the analysis. Far from being "only connections" the web is replete with structuring devices of different kinds. Far from standing independent of fixed hierarchical systems of organisation, the Web is situated within those more traditional structuring devices. These already existing structures do not preclude the possibility of complex interaction and emergence, but their role in that process is obscured by a simplistic appeal to emergence as outcome and not ongoing event. There are, then, fundamental problems with assuming the complexity of the web without exploring in adequate detail how complexity is manifested within the relationships between the many different structuring devices that also influence its evolution. Complexity theory was inculcated into the discourse of the web without this kind of critical engagement. On this level it can never be adequate to the kinds of broader social and cultural questions posed by the web. It cannot hope to do so because it refuses to engage with the processes that lead to the emergence of structure, choosing only to valorise the idea of emergence as an abstract ideal and

fait accompli. While

neither Berners-Lee nor O'Reilly claim to present fully developed sociological or epistemological models, drawing only on ideas with some currency within the computing subculture, it is precisely in its role as metaphor that the danger complexity theory in the discourse of the web emerges. The power and persuasiveness of the metaphor discourages analysis of both its underlying assumptions, and its realisation in the model of the web. It appropriates the rigour of complexity research without embodying that rigour. It validates its outlook by correlating the web with other systems that have been subject to extensive study, such as ecosystems, weather-systems, the social systems of insects, or evolutionary biology. In the process it avoids subjecting its own assumptions to full scrutiny. As the tacit political ideals of the computing subculture become overshadowed by the vehicle for their expression, lost in a metaphor that is inadequate to them, their real political force is disarmed. With the discourse of the web now converging on aWeb 3.0 model that also

\section{Complexity}

theory and

the web

\section{3}

borrows ideas from complexity in its valorisation of cloud computing, crowd-sourcing, total integration and ubiquitous computing, there is a need to engage both with the real social processes by which emergent structures form, and with their limitations and constraints. There is a need to replace the superficial metaphor of the web as mirror to both the complex society and complex mind with a critical investigation of the complex of complex systems into which the web is integrated, their entanglement, and their complicity.

Note

1. See Pastor-Satorras and Vespignani (2008)) for an example of an analogous attempt to understand the Internet and worldwide web in terms of general laws and concepts.

References

Anderson, N. (2006),

Tim Berners-Lee on Web 2.0: "Nobody Even Knows What It Means", available at: http://arstechnica.com/news.ars/post/20060901-7650.html (accessed: 6 October). Anderson, P.W. (1972), "More is different",

Science, Vol. 177 No. 4047, pp. 393-6.

Balkin, J.M. (1998),

Cultural Software: A Theory of Ideology, Yale University Press, New Haven, 
$\mathrm{CT}$ and London.

Berners-Lee, T. (1990),

Information Management: A Proposal, available at: www.w3.org/History/

1989/proposal.html (accessed 1 June 2006).

Berners-Lee, T. (1995),

Hypertext and our Collective Destiny, available at: www.w3.org/Talks/

9510_Bush/Talk.html (accessed: 20 June 2008).

Berners-Lee, T. (1999),

Weaving the Web: the Past, Present and Future of the Worldwide Web by

its Creator

, Orion Business Press, London.

Berners-Lee, T., Cailliau, R., Groff, J.-F. and Pollermann, B.D. (1992), "Worldwide web: the information universe",

Electronic Networking, Vol. 1 No. 1, pp. 52-8.

Blackmore, S. (1999),

The Meme Machine, Oxford University Press, Oxford and New York, NY.

Bush, V. (1945), "As we may think",

Atlantic Monthly, Vol. 176 No. 1, pp. 101-8.

Cilliers, P. (1998),

Complexity and Postmodernism: Understanding Complex Systems, Routledge,

London and New York, NY.

Cilliers, P. (2005), "Complexity, deconstruction and relativism",

Culture, Theory \& Society, Vol. 22

No. 5, pp. 255-67.

Dawkins, R. (1976),

The Selfish Gene, Oxford University Press, Oxford and New York, NY.

Dawkins, R. (1983),

The Extended Phenotype: The Long Reach of the Gene, Oxford University

Press, Oxford and New York, NY.

Dawkins, R. (1999), "Introduction”, in Blackmore, S. (Ed.),

The Meme Machine, Oxford

University Press, Oxford and New York, NY.

De Landa, M. (1997),

A Thousand Years of Nonlinear History, Zone Books, New York, NY.

Derrida, J. (1976) in Spivak, G.C. (trans.),

Of Grammatology, Johns Hopkins University Press,

Baltimore, MD and London.

DeveloperWorks (2006),

DeverloperWorks Interviews Tim Berners-Lee, available at: www-128.

ibm.com/developerworks/podcast/dwi/cm-int082206.html (accessed 7 August).

Ellis, D. (1992), "The physical and cognitive paradigms in information retrieval research",

Journal of Documentation

, Vol. 48 No. 1, pp. 45-64.

\section{JDOC}

\section{5,5}

\section{4}

Fikentscher, W. (1998),

Cultural Complexity, available at: www.santafe.edu/research/

publications/wpabstract/199810087 (accessed 1 August 2008).

Fodor, J. (1976),

The Language of Thought, Harvester Press, Hassocks.

Gardner, H. (1987),

The Mind's New Science: A History of the Cognitive Revolution; With a New

Epilogue by the Author

, Harper Collins, London.

Gillies, J. and Calliau, R. (2000),

How the Web was Born, Oxford University Press, Oxford and 
New York, NY.

Gladwell, M. (2002),

The Tipping Point: How Little Things Can Make a Big Difference, Abacus,

London.

Jakendoff, R. (2001), "Language in the ecology of the mind", in Cobley, P. (Ed.),

The Routledge

Companion to Semiotics and Linguistics

, Routledge, London and New York, NY, pp. 52-65.

Keen, A. (2007),

The Cult of the Amateur: How Today's Internet is Killing our Culture and

Assaulting Our Economy

, Nicholas Brealey Publishing, London and Boston, MA.

Landow, G.P. (1997),

Hypertext 2.0: The Convergence of Contemporary Critical Theory and

Technology

, John Hopkins University Press, Baltimore, MD and London.

Lewin, R. (1999),

Complexity: Life at the Edge of Chaos, 2nd ed., University of Chicago Press,

Chicago, IL.

McKnight, C., Dillon, A. and Richardson, J. (1991),

Hypertext in Context, Cambridge University

Press, Cambridge.

Manson, S.M. (2000), "Simplifying complexity: a review of complexity theory",

Geoform, Vol. 32,

pp. 405-14.

Miller, P. (2005), "Web 2.0: building the new library",

Ariadne 45, available at: www.ariadne.ac.

uk/issue 45/miller/ (accessed: 26 June 2008).

Naughton, J. (1999),

A Brief History of the Future: the Origins of the Internet, Weidenfield \&

Nicolson, London.

Nowotny, H. (2005), "The increase of complexity and its reduction",

Culture, Theory \& Society,

Vol. 22 No. 5, pp. 15-31.

O'Reilly, T. (2005),

What is Web 2.0?, available at: www.oreillynet.com/pub/a/oreilly/tim/news/

2005/09/30/what-is-Web-20.html?page

L'1 (accessed: 20 October 2007).

Paolillo, J.

et al. (2005), Measuring Linguistic Diversity on the Internet, United Nations Education, Scientific and Cultural Organisation, Paris, available at: http://unesdoc.unesco.org/images/ 0014/001421/142186e.pdf (accessed: 18 October 2007).

Pastor-Satorras, R. and Vespignani, A. (2008),

The Evolution and Structure of the Internet:

A Statistical Physics Approach

, Cambridge University Press, Cambridge.

Penrose, R. (1989),

The Emperor's New Mind: Concerning Computers, Minds, and the Laws of

Physics

, Oxford University Press, Oxford and New York, NY.

Penrose, R. (1989),

Shadows of the Mind: Searching for the Missing Science of Consciousness, new

ed., Vintage, London.

Pinker, S. (1994),

The Language Instinct: The New Science of Language and Mind, Allan Lane,

London.

Pinker, S. (1997),

How the Mind Works, Allen Lane, London.

Roszak, T. (1988), 
The Cult of Information: The Folklore of Computing and the True Art of Thinking

, Paladin, London.

Shaw, R. (2005),

Web 2.0? It Doesn't Exist, available at: http://blogs.zdnet.com/ip-telephony/ ?p

L'805 (accessed 7 August 2007).

\section{Complexity}

\section{theory and}

\section{the web}

\section{5}

Sterling, B. (2005), "Order out of chaos",

Wired, Vol. 13 4, April, available at: www.wired.com/

wired/archive/13.04/view.html?pg

L4 (accessed 30 June 2008).

Surowiecki, J. (2004),

The Wisdom of Crowds: Why the Many are Smarter than the Few and How Collective Wisdom Shapes Business, Economies, Societies and Nations

, Little Brown,

London.

Taylor, M.C. (2001),

The Moment of Complexity: Emerging Network Culture, University of

Chicago Press, Chicago, IL and London.

Thrift, N. (1999), "The place of complexity",

Theory, Culture and Society, Vol. 16 No. 3, pp. 31-69.

Tredinnick, L. (2006a),

Digital Information Contexts: Theoretical Approaches to Understanding

Digital Information

, Chandos Publishing, Oxford.

Tredinnick, L. (2006b), "Web 2.0 and business: a pointer to the intranets of the future?",

Business

Information Review

, Vol. 23 No. 4, pp. 226-34.

Tredinnick, L. (2006c), "Anarchy and the organisation: Intranet 2.0",

Online Information

Proceedings

, 27-29 November, pp. 163-7.

Tredinnick, L. (2008),

Digital Information Culture: The Individual and Society in the Digital Age, Chandos Publishing, Oxford.

Urry, J.N. (2005), "The complexity turn",

Theory, Culture \& Society, Vol. 22 No. 5, pp. 1-14.

Vander Wal, T. (2007),

Folksonomy, available at: http://vanderwal.net/folksonomy.html (accessed

30 June 2008)

von Baeyer, H.C. (2003),

Information: the New Language of Science, Weidenfield \& Nicolson,

London.

Waldrop, M.M. (1992),

Complexity: The Emerging Science at the Edge of Order and Chaos, Simon

\& Schuster, New York, NY.

Woodhead, N. (1991),

Hypertext and Hypermedia: Theory and Applications, Sigma Press,

Wilmslow.

Further reading

Tredinnick, L. (2004), 
Why Intranets Fail (and How to Fix Them), Chandos Publishing, Oxford. Vander Wal, T. (2005a),

Explaining and Showing Broad and Narrow Folksonomies, available at: www.vanderwal.net/random/entrysel.php?blog

L'1635 (accessed 27 June 2008).

Vander Wal, T. (2005b),

Designing with a Solution is the Problem, available at: www.vanderwal. net/random/entrysel.php?blog

L'1687 (accessed 27 June 2008).

Corresponding author

Luke Tredinnick can be contacted at: 1.tredinnick@londonmet.ac.uk

\section{JDOC}

\section{5,5}

\section{6}

To purchase reprints of this article please e-mail:

reprints@emeraldinsight.com

Or visit our web site for further details:

www.emeraldinsight.com/reprints 